\title{
Commentary Clinical trial update: International Breast Cancer Study Group
} Karen N Price ${ }^{1}$ and Aron Goldhirsch ${ }^{2,3}$ for the International Breast Cancer Study Group

\author{
${ }^{1}$ International Breast Cancer Study Group Statistical Center, Frontier Science and Technology Research Foundation, Boston, Massachusetts, USA \\ 2Oncology Institute of Southern Switzerland, Bellinzona, Mendrisio, Lugano, Switzerland \\ ${ }^{3}$ European Institute of Oncology, Milan, Italy
}

Corresponding author: Karen N Price, price@jimmy.harvard.edu

Published: 4 October 2005

This article is online at http://breast-cancer-research.com/content/7/6/252

(c) 2005 BioMed Central Ltd
Breast Cancer Research 2005, 7:252-254 (DOI 10.1186/bcr1334)

breast cancer (i.e. estrogen receptor-positive and/or progesterone receptor-positive) who were included in trials of chemotherapy and of endocrine therapy across the board. Through subgroup analyses of these large heterogeneous trials we have identified distinct responsiveness features, and the current generation of IBCSG trials investigates treatments tailored to populations based on these features (Table 1). Examples of such trials are described below.

(a) specific subpopulations of patients wi breast cancer, believing that what is best for the majority may not be best for a defined minority.

\section{Introduction}

The International Breast Cancer Study Group (IBCSG) opened its first generation of trials in 1978. The IBCSG includes both participating cooperative groups and individual institutions from all over the world. The groups are from Switzerland (Swiss Group for Clinical Cancer Research, 38 centers), Sweden (West Sweden Breast Cancer Study Group, 11 centers), Australia and New Zealand (Australian New Zealand Breast Cancer Trials Group, 56 centers), and Chile (GOCCHI, a Chilean cooperative group, 23 centers). Individual centers are located in Italy (14 centers), Slovenia, Hungary (2 centers), Spain, Romania, Austria, United Kingdom, Belgium (2 centers), Brazil, Peru, Hong Kong, India, South Africa (2 centers), and Canada.

The IBCSG is conducting trials of tailored treatment approaches for these subpopulations: patients with endocrine non-responsive early breast cancer; older patients with endocrine non-responsive early breast cancer who are not candidates for standard chemotherapy regimens; and younger patients with endocrine responsive early breast cancer. Because each of these three populations is somewhat rare, treatment decisions tend to be based on the findings from the largest breast cancer population: middleaged (median age 55) women with endocrine responsive

\section{Tailored chemotherapy trial for women with endocrine non-responsive breast cancer: the CM-Maintenance trial}

The CM-Maintenance Trial (IBCSG 22-00) studies a tailored chemotherapy approach for patients with endocrine nonresponsive tumors. The role of prolonged, low-dose chemotherapy after a standard adjuvant chemotherapy regimen to reduce the risk of relapse and improve survival is being investigated. Unlike patients with endocrine responsive disease, who benefit from five years of endocrine therapy after chemotherapy, there is no opportunity for a useful prolonged treatment for patients with endocrine non-responsive disease beyond a standard duration (six months). The low-dose chemotherapy (metronomic) regimen used in this trial (cyclophosphamide and methotrexate (CM) given orally) was developed in advanced disease and resulted in tumor shrinkage even in patients heavily pretreated [1]. This combination of drugs might be effective by damaging the tumors' vessels (antiangiogenesis) or by interfering with progression of the stromal structure of metastases. In the trial, one year of CM is compared with no further therapy beyond the standard adjuvant program. This ongoing trial was designed to allow a new drug with proven anti-angiogenic effects, if one becomes available, to replace the current metronomic regimen $(\mathrm{CM})$.

As of June 30, 2005, 339 of the required 900 patients have been enrolled from 22 participating centers. Accrual to the 
Table 1

\begin{tabular}{|c|c|c|c|c|}
\hline IBCSG Trial & Population & Activation & Target accrual & Comparison(s) \\
\hline $\begin{array}{l}\text { CM-maintenance } \\
\text { IBCSG 22-00 }\end{array}$ & Endocrine non-responsive & November 2000 & 900 & $\begin{array}{l}\text { Induction CT versus } \\
\text { Induction } \mathrm{CT} \rightarrow \mathrm{CM} \\
\text { maintenance }\end{array}$ \\
\hline $\begin{array}{l}\text { CASA } \\
\text { IBCSG 32-05 }\end{array}$ & $\begin{array}{l}\text { Endocrine non-responsive, } \\
\text { older, not suitable for } \\
\text { standard CT }\end{array}$ & August 2005 & 1,296 & $\begin{array}{l}\text { Option 1: PLD versus nil } \\
\text { Option 2: PLD versus low } \\
\text { dose CM }\end{array}$ \\
\hline $\begin{array}{l}\text { SOFT } \\
\text { IBCSG 24-02 }\end{array}$ & $\begin{array}{l}\text { Premenopausal at } \\
\text { randomization }\end{array}$ & August 2003 & 3,000 & $\begin{array}{l}\text { Tamoxifen alone versus } \\
\text { OFS }+ \text { tamoxifen versus } \\
\text { OFS + exemestane }\end{array}$ \\
\hline $\begin{array}{l}\text { TEXT } \\
\text { IBCSG 25-02 }\end{array}$ & Should get OFS at start & August 2003 & 1,845 & $\begin{array}{l}\text { Triptorelin } \pm \mathrm{CT}+\text { tamoxifen } \\
\text { versus triptorelin } \pm \mathrm{CT}+ \\
\text { exemestane }\end{array}$ \\
\hline $\begin{array}{l}\text { PERCHE } \\
\text { IBCSG 26-02 }\end{array}$ & $\begin{array}{l}\text { Should get OFS at start, } \\
\text { lower risk }\end{array}$ & August 2003 & 1,750 & $\begin{array}{l}\text { OFS }+ \text { (tamoxifen or } \\
\text { exemestane) versus OFS }+ \\
\text { CT }+(\text { tamoxifen or } \\
\text { exemestane) }\end{array}$ \\
\hline
\end{tabular}

$\mathrm{CM}$, cyclophosphamide and methotrexate; CT = chemotherapy; IBCSG = International Breast Cancer Study Group; OFS = ovarian function suppression; PLD = pegylated liposomal doxorubicin.

trial has been slower than anticipated, and the IBCSG is actively seeking new participants.

\section{Tailored chemotherapy trial for women at advanced age with endocrine non-responsive breast cancer: the CASA trial}

Aging remains one of the single greatest risk factors for the development of new breast cancer, with the estimated risk of new breast cancer at 1 in 14 for women aged 60 to 79 years compared with 1 in 24 women aged 40 to 59 years and 1 in 228 women aged 39 years and younger [2]. In western countries, approximately $50 \%$ of women with breast cancer are 65 years of age or older [3]. Because breast cancer incidence increases with age, more cases can be expected among elderly women [3]. Despite this fact, the appropriate use of adjuvant chemotherapy for elderly women with breast carcinoma remains unknown, especially for women with an endocrine non-responsive early breast cancer who have no opportunity to be treated for reduction of risk of relapse with hormonal therapies. Even without considering endocrine responsiveness, efficacy data in women aged $>70$ years are scarce, resulting in a lack of clear guidelines for patients in this age group. In the 2005 Overview Analysis [4], which combined data from most of the clinical trials for patients with early breast cancer, only $4 \%$ of the women enrolled in trials with a comparison of multi-agent chemotherapy to nil were aged 70 or older.

To accommodate physician and patient preferences, the IBCSG has designed two complementary randomization options within one clinical trial, Trial 32-05 (CASA), to investigate the role of adjuvant cytotoxic chemotherapy for women at advanced age with endocrine non-responsive early breast cancer who are not suitable for one of the standard chemotherapy regimens. Pegylated liposomal doxorubicin (PLD) is the experimental treatment. For each eligible patient, the investigator will choose one of the two randomization options based on physician and/or patient preference. Option 1, CASA-nil: PLD versus nil, for patients who are candidates to receive no adjuvant therapy. Option 2, CASA-CM: PLD versus $\mathrm{CM}$, for patients who should receive some adjuvant treatment.

The primary evaluation will be to compare PLD versus nonPLD-containing regimens in the pooled analysis of both pairwise comparisons.

The CASA trial was distributed in August 2005 for participating center approval and activation. The total required sample size is 1,296 patients.

\section{Tailored treatment investigations for premenopausal women with endocrine responsive breast cancer: the SOFT, TEXT, and PERCHE (STP) trials}

The STP trials are complementary studies designed to answer three open questions concerning adjuvant treatment for premenopausal patients with endocrine responsive disease: What is the role of ovarian function ablation/ suppression (OFS)? What is the role of aromatase inhibitors? What is the role of chemotherapy? We know that chemotherapy, tamoxifen and OFS are individually effective adjuvant treatment modalities in women under 50 years of age with estrogen receptor-positive breast cancer $[5,6]$. The addition 
of 5 years of tamoxifen to adjuvant chemotherapy in this group results in an additional approximately $40 \%$ reduction in the odds of recurrence or death $[7,8]$. These data suggest that adjuvant combination chemo-endocrine strategies can improve results over single modality treatments.

Conclusive data on the best approaches to treat this population are scarce because positive results for chemotherapy in the 1970s (mainly for women in their forties) and misleading overview reports on tamoxifen (claimed to be ineffective in younger women) in the 1980s and 1990s, inhibited evaluation of endocrine therapies for younger women. Adjuvant treatment of choice for individual premenopausal women with endocrine responsive disease is largely based upon assessment of risk of relapse and physician's perception on the relative role of endocrine versus cytotoxic approaches. To offer a randomized clinical trial to define a better adjuvant treatment for premenopausal women with endocrine responsive disease, three tailored treatment investigations are being conducted globally by the IBCSG through the Breast International Group and the North American Breast Intergroup.

The Suppression of Ovarian Function Trial (SOFT: IBCSG 24-02) is designed to determine the role of OFS and the role of aromatase inhibitors for women who remain premenopausal after surgery alone or after completion of adjuvant and/or neoadjuvant chemotherapy.

The Tamoxifen and Exemestane Trial (TEXT: IBCSG 25-02) is designed to determine the role of aromatase inhibitors for women who receive OFS (with triptorelin) from the start of adjuvant therapy. Chemotherapy, if given, should be started with the triptorelin and followed by the tamoxifen or exemestane. Use of chemotherapy is by investigator/patient choice or by randomized assignment in the PERCHE trial.

The Premenopausal Endocrine Responsive Chemotherapy Trial (PERCHE: IBCSG 26-02) is designed to determine the role of chemotherapy. It features randomization either to OFS plus tamoxifen or exemestane, or to chemotherapy plus OFS plus tamoxifen or exemestane. Women for whom the role of adding chemotherapy to 'complete estrogen blockade' is uncertain should be offered PERCHE.

As of June 30, 2005, 164 participating centers from North America, Europe, Australia, South America, and Africa have randomized patients on these trials, including 243 on SOFT, 432 on TEXT, and 10 on PERCHE. The total required sample size is $3,000,1,845$ and 1,750 patients, respectively.

\section{Translational research in CASA, CM-maintenance, and STP}

All of the trials include collection of tissue blocks (both tumor and normal) for translational research. Central laboratory (immunohistochemical percent stained cells), HER-2/neu, Ki67, grade, and histological type are planned. Translational research projects proposed by scientists among IBCSG members or outside the Group are encouraged. Definition of new subgroups of patients within the endocrine nonresponsive population of the CASA trial, differential responsiveness patterns to tamoxifen or to exemestane by hormone receptors and HER-2/neu for patients in the STP trials, and the evaluation of several angiogenesis and stromal markers in the CM-Maintenance trial are ongoing or planned. This translational research will provide the scientific foundation for the therapeutic trials conducted in specific populations.

\section{Competing interests}

The International Breast Cancer Study Group receives central funding for drug supply and trial conduct from Schering Plough for the CASA trial and from Pfizer for the STP trials. The authors declare they have no competing interests.

\section{Acknowledgements}

We thank the patients, physicians, nurses, and data managers who participate in the International Breast Cancer Study Group trials. Additional support for central coordination, data management, and statistics provided by the Swedish Cancer Society, The Cancer Council Australia, Australian New Zealand Breast Cancer Trials Group (NHMRC grants), the Frontier Science and Technology Research Foundation, the Swiss Group for Clinical Cancer Research (SAKK), and the United States National Cancer Institute (CA-75362). We also acknowledge support for the Cape Town participants from the Cancer Association of South Africa, and for the St Gallen participants from the Foundation for Clinical Cancer Research of Eastern Switzerland.

\section{References}

1. Colleoni M, Rocca A, Sandri MT, Zorzino L, Masci G, Nole F, Peruzzotti G, Robertson C, Orlando L, Cinieri S, et al.: Low-dose oral methotrexate and cyclophosphamide in metastatic breast cancer: antitumor activity and correlation with vascular endothelial growth factor levels. Ann Oncol 2002, 13:73-80.

2. Jemal A, Murray T, Samuels A, Ghafoor A, Ward E, Thun MJ: Cancer statistics, 2003. CA Cancer J Clin 2003, 53:5-26.

3. Crivellari D, Price K, Gelber RD, Castiglione-Gertsch M, Rudenstam CM, Lindtner J, Fey MF, Senn HJ, Coates AS, Collins J, Goldhirsch A: International Breast Cancer Study Group. Adjuvant endocrine therapy compared with no systemic therapy for elderly women with early breast cancer: 21 -year results of International Breast Cancer Study Group Trial IV. J Clin Oncol 2003, 21:4517-4523.

4. Early Breast Cancer Trialists' Collaborative Group (EBCTCG): Effect of chemotherapy and hormonal therapy for early breast cancer on recurrence and 15-year survival: an overview of the randomised trials. Lancet 2005, 365:1687-1717.

5. Early Breast Cancer Trialists' Collaborative Group: Effects of adjuvant tamoxifen and of cytotoxic therapy on mortality in early breast cancer: an overview of 61 randomized trials among 28,896 women. New Engl J Med 1988, 319:1681-1692.

6. Early Breast Cancer Trialists' Collaborative Group: Systemic treatment of early breast cancer by hormonal, cytotoxic or immune therapy: 133 randomized trials involving 31,000 recurrences and 24,000 deaths among 75,000 women. Lancet 1992, 339:1-15 and 71-85.

7. Early Breast Cancer Trialists' Collaborative Group: Tamoxifen for early breast cancer: an overview of the randomised trials. Lancet 1998, 351:1451- 1467.

8. Fisher B, Dignam J, Wolmark N, DeCillis A, Emir B, Wickerham DL, Bryant J, Dimitrov NV, Abramson N, Atkins JN, et al.: Tamoxifen and chemotherapy for lymph node negative, estrogen receptor-positive breast cancer. J Natl Cancer Inst 1997, 89:1673-1682. 